\title{
Research Article \\ Orlicz Sequence Spaces with a Unique Spreading Model
}

\author{
Cuixia Hao, ${ }^{1}$ Linlin L $\ddot{u_{1}}{ }^{2}$ and Hongping Yin ${ }^{3}$ \\ ${ }^{1}$ Department of Mathematics, Heilongjiang University, Harbin 150080, China \\ ${ }^{2}$ Department of Information Science, Star College of Harbin Normal University, Harbin 150025, China \\ ${ }^{3}$ Department of Mathematics, Inner Mongolia University, Tongliao 028000, China
}

Correspondence should be addressed to Cuixia Hao, haocuixia@yahoo.com

Received 24 December 2009; Accepted 23 March 2010

Academic Editor: Shusen Ding

Copyright $\odot 2010$ Cuixia Hao et al. This is an open access article distributed under the Creative Commons Attribution License, which permits unrestricted use, distribution, and reproduction in any medium, provided the original work is properly cited.

We study the set of all spreading models generated by weakly null sequences in Orlicz sequence spaces equipped with partial order by domination. A sufficient and necessary condition for the above-mentioned set whose cardinality is equal to one is obtained.

\section{Introduction}

Let $X$ be a separable infinite dimensional real Banach space. There are three general types of questions we often ask. In general, not much can be said in regard to this question "what can be said about the structure of $X$ itself" and not much more can be said about the question "does $X$ embedded into a nice subspace". The source of the research on spreading models was mainly from the question "finding a nice subspace $Y \subseteq X$ " [1]. The spreading models usually have a simpler and better structure than the class of subspaces of $X[2,3]$. In this paper, we study the question concerning the set of all spreading models whose cardinality is equal to one.

The notion of a spreading model is one of the application of Ramsey theory. It is a useful tool of digging asymptotic structure of Banach space, and it is a class of asymptotic unconditional basis. In 1974, Brunel and Sucheston [4] introduced the concept of spreading model and gave a result that every normalized weakly null sequence contains an asymptotic unconditional subsequence, they call the subsequence spreading model. It was not until the last ten years that the theory of spreading models was developed, especially in recent five years. In 2005, Androulakis et al. in [2] put forward several questions on spreading models and solved some of them. Afterwards, Sari et al. discussed some problems among them and obtained fruitful results. This paper is mainly motivated by some results obtained by Sari et al. in their papers $[3,5]$. 


\section{Preliminaries and Observations}

An Orlicz function $M$ is a real-valued continuous nondecreasing and convex function defined for $t \geq 0$ such that $M(0)=0$ and $\lim _{t \rightarrow \infty} M(t)=\infty$. If $M(t)=0$ for some $t>0, M$ is said to be a degenerate function. $M(u)$ is said to satisfy the $\Delta_{2}$ condition $\left(M \in \Delta_{2}\right)$ if there exist $K$, $u_{0}>0$ such that $M(2 u) \leq K M(u)$ for $0 \leq u \leq u_{0}$. We denote the modular of a sequence of numbers $x=\{x(i)\}_{i=1}^{\infty}$ by $\rho_{M}(x)=\sum_{i=1}^{\infty} M(x(i))$. It is well known that the space

$$
l_{M}=\left\{x=\{x(i)\}_{i=1}^{\infty}: \rho_{M}(\lambda x)=\sum_{i=1}^{\infty} M(\lambda x(i))<\infty \text { for some } \lambda>0\right\}
$$

endowed with the Luxemburg norm

$$
\|x\|=\inf \left\{\lambda>0: \rho_{M}\left(\frac{x}{\lambda}\right) \leq 1\right\}
$$

is a Banach sequence space which is called Orlicz sequence space. The space

$$
h_{M}=\left\{x=\{x(i)\}: \rho_{M}(\lambda x)=\sum_{i=1}^{\infty} M(\lambda x(i))<\infty \text { for each } \lambda>0\right\}
$$

is a closed subspace of $l_{M}$. It is easy to verify that the spaces $l^{p}(1 \leq p<\infty)$ are just Orlicz sequence spaces, and Orlicz sequence spaces are the generalization of the spaces $l^{p}(1 \leq p<$ $\infty)$. Furthermore, if $M$ is a degenerate Orlicz function, then $l_{M} \cong l_{\infty}$ and $h_{M} \cong c_{0}$ [6]. In the context, the Orlicz functions considered are nondegenerate. Let

$$
E_{M, 1}=\overline{\left\{\frac{M(\lambda t)}{M(\lambda)}: 0<\lambda<1\right\}}, \quad C_{M, 1}=\overline{\operatorname{conv}} E_{M, 1}
$$

They are nonvoid norm compact subsets of $C(0,1)$ consisting entirely of Orlicz functions which might be degenerate [6, lemma 4.a.6].

Definition 2.1. Let $X$ be a separable infinite dimensional Banach space. For every normalized basic sequence $\left(y_{i}\right)$ in a Banach space and for every $\varepsilon_{n} \downarrow 0$, there exist a subsequence $\left(x_{i}\right)$ and a normalized basic sequence $\left(\tilde{x}_{i}\right)$ such that for all $n \in N,\left(a_{i}\right)_{i=1}^{n} \in[-1,1]^{n}$ and $n \leq k_{1}<\cdots<k_{n}$,

$$
\left\|\sum_{i=1}^{n} a_{i} x_{k_{i}}\right\|-\left\|\sum_{i=1}^{n} a_{i} \tilde{x}_{i}\right\| \mid<\varepsilon_{n} .
$$

The sequence $\left(\tilde{x}_{i}\right)$ is called the spreading model of $\left(x_{i}\right)$ and it is a suppression- 1 unconditional basic sequence if $\left(y_{i}\right)$ is weakly null [4].

The following theorem guarantees the existence of a spreading model of $X$. We shall give a detailed proof. 
Theorem 2.2. Let $\left(x_{n}\right)$ be a normalized basic sequence in $X$ and let $\varepsilon_{n} \downarrow 0$. Then there exists a subsequence $\left(y_{n}\right)$ of $\left(x_{n}\right)$ so that for all $n,\left(a_{i}\right)_{i=1}^{n} \subseteq[-1,1]$ and integers $n \leq k_{1}<k_{2}<\cdots k_{n}, n \leq$ $i_{1}<i_{2}<\cdots i_{n}$

$$
\left\|\left|\sum_{j=1}^{n} a_{j} y_{k_{j}}\|-\| \sum_{j=1}^{n} a_{j} y_{i_{j}} \|\right|<\varepsilon_{n} .\right.
$$

In order to prove Theorem 2.2, we should have to recall the following definitions and theorem.

For $k \in N,[N]^{k}$ is the set of all subsets of $N$ of size $k$. We may take it as the set subsequences of length $k,\left(n_{i}\right)_{i=1}^{k}$ with $n_{1}<\cdots<n_{k}$. [N] $]^{\omega}$ denotes all subsequences of $N$. Similar definitions apply to $[M]^{k}$ and $[M]^{w}$ if $M \in[N]^{w}$.

Definition 2.3 (see [1]). Let $I_{1}$ and $I_{2}$ be two disjoint intervals. For any $\left(k_{1}, \ldots, k_{n}\right),\left(i_{1}, \ldots, i_{n}\right) \in$ $[N]^{k}$ and scalars $\left(a_{i}\right)_{i=1}^{n}$ if

$$
\left\|\sum_{j=1}^{n} a_{j} y_{k_{j}}\right\| \in I_{i}, \quad\left\|\sum_{j=1}^{n} a_{j} y_{i_{j}}\right\| \in I_{i} \quad(i=1 \text { or } 2),
$$

then we call $I_{i}$ ( $i=1$ or 2$)$ "color" $\left(k_{1}, \ldots, k_{n}\right)$ and $\left(i_{1}, \ldots, i_{n}\right)$. Meanwhile, we say $\left(k_{1}, \ldots, k_{n}\right)$ has the same "color" as $\left(i_{1}, i_{2}, \ldots, i_{n}\right)$, where $\left(y_{i}\right)$ is a sequence of a Banach space. We identify the same "color" subsets of $[N]^{k}$, saying they are 1-colored.

Definition 2.4 (see [1]). The family of $[N]^{k}(k \in N)$ is called finitely colored provided that it only contains finite subsets in "color" sense, and each subset is 1-colored.

Theorem 2.5 (see [1]). Let $k \in N$ and let $[N]^{k}$ be finitely colored. Then there exists $M \in[N]^{\omega}$ so that $[M]^{k}$ is 1-colored.

Proof of Theorem 2.2. We accomplish the proof in two steps.

Step 1. We shall prove that for any $n \in Z^{+}$, there exists $\left(y_{i}\right) \subseteq\left(x_{i}\right)$ such that for any $\left(a_{i}\right)_{i=1}^{n} \subseteq$ $[-1,1], n \leq k_{1}<k_{2}<\cdots k_{n}, n \leq i_{1}<i_{2}<\cdots i_{n}$,

$$
\left\||| \sum_{j=1}^{n} a_{j} y_{k_{j}}\right\|-\left\|\sum_{j=1}^{n} a_{j} y_{i_{j}}\right\| \mid<\varepsilon_{n}(*) .
$$

Firstly, for fixed $\left(a_{i}\right)_{i=1}^{n} \subseteq[-1,1]$, by the Definition 2.4, we can prove that the above inequality holds. In fact, we partition $[0, n]$ into subintervals $\left(I_{j}\right)_{j=1}^{m}$ of length $<\varepsilon_{n}$ and "color" 
$\left(k_{1}, k_{2}, \ldots k_{n}\right)$ by $I_{l}$ if

$$
\left\|\sum_{j=1}^{n} a_{j} y_{k_{j}}\right\| \in I_{l}
$$

In the same way, we can also "color" $\left(i_{1}, i_{2}, \cdots i_{n}\right)$ by $I_{l}$.

We can take $[-1,1]^{n}$ as the unit ball in finite-dimensional space $l_{1}^{n}$; then $[-1,1]^{n}$ is sequentially compact; moreover, it is totally bounded and complete. Under $l_{1}^{n}$-metric, take $N=\left\{z_{1}^{(n)}, z_{2}^{(n)}, \ldots z_{m}^{(n)}\right\}$ for $\left(\varepsilon_{n} / 4\right)$-net of $[-1,1]^{n}$. For any element of net $N$, repeat the above process, and let $z_{k}^{(n)}=\left(z_{k_{j}}^{(n)}\right)_{j=1}^{n}, k=1,2, \ldots m$. We partition $[0, n]$ into subintervals $\left(I_{l}\right)_{l=1}^{m}$ of length $<\varepsilon_{n} / 2$ and "color" $\left(k_{1}, k_{2}, \ldots, k_{n}\right)$ by $I_{l}$ if

$$
\left\|\sum_{j=1}^{n} z_{k_{j}}^{(n)} y_{i_{j}}\right\| \in I_{l}
$$

Since the length of $I_{l}<\varepsilon_{n} / 2$, we have

$$
\left\||| \sum_{j=1}^{n} z_{k_{j}}^{(n)} y_{k_{j}}\right\|-\left\|\sum_{j=1}^{n} z_{k_{j}}^{(n)} y_{i_{j}}\right\| \mid<\frac{\varepsilon_{n}}{2} \quad(k=1,2, \ldots, m) .
$$

Secondly, we shall prove that for any $\left(a_{i}\right)_{i=1}^{n} \subseteq[-1,1]^{n},(*)$ holds. Since $N=\left\{z_{1}^{(n)}, z_{2}^{(n)}, \ldots z_{m}^{(n)}\right\}$ is the $\left(\varepsilon_{n} / 4\right)$-net of $[-1,1]^{n}$, there exists $z_{k_{0}}^{(n)}=\left(z_{k_{0_{j}}}^{(n)}\right)_{j=1}^{n}$ such that

$$
\left\|\left(a_{i}\right)_{i=1}^{n}-z_{k_{0}}^{(n)}\right\|=\sum_{j=1}^{n}\left|a_{j}-z_{k_{0 j}}^{(n)}\right|<\frac{\varepsilon_{n}}{4} .
$$

Therefore, we have

$$
\begin{aligned}
\left\|\sum_{j=1}^{n} a_{j} y_{k_{j}}\right\| & \leq\left\|\sum_{j=1}^{n}\left(a_{j}-z_{k_{0_{j}}}^{(n)}\right) y_{k_{j}}\right\|+\left\|\sum_{j=1}^{n} z_{k_{0_{j}}}^{(n)} y_{k_{j}}\right\| \\
& \leq \sum_{j=1}^{n}\left|a_{j}-z_{k_{0_{j}}}^{(n)}\right| \cdot\left\|y_{k_{j}}\right\|+\left\|\sum_{j=1}^{n} z_{k_{0_{j}}}^{(n)} y_{k_{j}}\right\| \\
& =\sum_{j=1}^{n}\left|a_{j}-z_{k_{0_{j}}}^{(n)}\right|+\left\|\sum_{j=1}^{n} z_{k_{0_{j}}}^{(n)} y_{k_{j}}\right\| \\
& <\frac{\varepsilon_{n}}{4}+\left\|\sum_{j=1}^{n} z_{k_{0_{j}}}^{(n)} y_{k_{j}}\right\| .
\end{aligned}
$$


Hence,

$$
||\left|\sum_{j=1}^{n} a_{j} y_{k_{j}}\|-\| \sum_{j=1}^{n} z_{k_{0_{j}}}^{(n)} y_{k_{j}} \|\right|<\frac{\varepsilon_{n}}{4} .
$$

Similarly, we obtain

$$
\left\||| \sum_{j=1}^{n} a_{j} y_{i_{j}}\right\|-\left\|\sum_{j=1}^{n} z_{k_{0_{j}}}^{(n)} y_{i_{j}}\right\| \mid<\frac{\varepsilon_{n}}{4} .
$$

Thus

$$
\begin{aligned}
& \|\| \sum_{j=1}^{n} a_{j} y_{k_{j}}\|-\| \sum_{j=1}^{n} a_{j} y_{i_{j}} \| \mid \\
& =\left|\left\|\sum_{j=1}^{n} a_{j} y_{k_{j}}\right\|-\left\|\sum_{j=1}^{n} z_{k_{0_{j}}}^{(n)} y_{k_{j}}\right\|+\left\|\sum_{j=1}^{n} z_{k_{0_{j}}}^{(n)} y_{k_{j}}\right\|-\left\|\sum_{j=1}^{n} a_{j} y_{i_{j}}\right\|+\left\|\sum_{j=1}^{n} z_{k_{0_{j}}}^{(n)} y_{i_{j}}\right\|-\left\|\sum_{j=1}^{n} z_{k_{0_{j}}}^{(n)} y_{i_{j}}\right\|\right| \\
& \leq\left|\left\|\sum_{j=1}^{n} a_{j} y_{k_{j}}\right\|-\left\|\sum_{j=1}^{n} z_{k_{0_{j}}}^{(n)} y_{k_{j}}\right\|\right|+\left\|\sum_{j=1}^{n} z_{k_{0_{j}}}^{(n)} y_{k_{j}}\right\|-\left\|\sum_{j=1}^{n} a_{j} y_{i_{j}}\right\||| \\
& \quad+\mid\left\|\sum_{j=1}^{n} z_{k_{0_{j}}}^{(n)} y_{k_{j}}\right\|-\left\|\sum_{j=1}^{n} z_{k_{0_{j}}}^{(n)} y_{i_{j}}\right\| \\
& <\frac{\varepsilon_{n}}{4}+\frac{\varepsilon_{n}}{4}+\frac{\varepsilon_{n}}{2}=\varepsilon_{n} .
\end{aligned}
$$

Step 2. We apply diagonal argument to prove that there exists $\left(y_{i}\right) \subseteq\left(x_{i}\right)$ such that for any $n \in Z^{+},\left(a_{i}\right)_{i=1}^{n} \subseteq[-1,1], n \leq k_{1}<k_{2}<\cdots k_{n}, n \leq i_{1}<i_{2}<\cdots i_{n}$,

$$
\left|\left\|\sum_{j=1}^{n} a_{j} y_{k_{j}}\right\|-\left\|\sum_{j=1}^{n} a_{j} y_{i_{j}}\right\|\right|<\varepsilon_{n} .
$$

By Step 1, in view of $n=1$, there exists $\left(y_{i}^{(1)}\right) \subseteq\left(x_{i}\right)$ such that for any $a \in[-1,1]$, for any $k_{1} \in Z^{+}, i_{1} \in Z^{+}, n \leq k_{1}, n \leq i_{1}$, we have

$$
\left|\left\|a y_{k_{1}}^{(1)}\right\|-\left\|a y_{i_{1}}^{(1)}\right\|\right|<\varepsilon_{1} .
$$


Obviously, $\left\{y_{i}^{(1)}\right\}$ is also a normalized basic sequence. So in view of $n=2$, there exists $\left(y_{i}^{(2)}\right) \subseteq$ $\left(y_{i}^{(1)}\right)$ such that for any $\left(a_{i}\right)_{i=1}^{2} \subseteq[-1,1], n \leq k_{1}<k_{2}, n \leq i_{1}<i_{2}$,

$$
\left\|\left|\sum_{j=1}^{2} a_{j} y_{k_{j}}^{(2)}\|-\| \sum_{j=1}^{2} a_{j} y_{i_{j}}^{(2)} \|\right|<\varepsilon_{2} .\right.
$$

Repeating the above process, for any $n$, there exists $\left(y_{i}^{(n)}\right) \subseteq\left(y_{i}^{(n-1)}\right)$ such that for any $\left(a_{i}\right)_{i=1}^{n} \subseteq$ $[-1,1], n \leq k_{1}<k_{2}<\cdots k_{n}, n \leq i_{1}<i_{2}<\cdots i_{n}$, we have

$$
\left\||| \sum_{j=1}^{n} a_{j} y_{k_{j}}^{(n)}\right\|-\left\|\sum_{j=1}^{n} a_{j} y_{i_{j}}^{(n)}\right\| \mid<\varepsilon_{n} .
$$

Finally, we choose the diagonal subsequence $\left(y_{i}^{(i)}\right) \subset\left(x_{i}\right)$; for any $n,\left(a_{i}\right)_{i=1}^{n} \subseteq[-1,1], n \leq k_{1}<$ $k_{2}<\cdots k_{n}, n \leq i_{1}<i_{2}<\cdots i_{n}$, we obtain that

$$
||\left|\sum_{j=1}^{n} a_{j} y_{k_{j}}^{\left(k_{j}\right)}\|-\| \sum_{j=1}^{n} a_{j} y_{i_{j}}^{\left(i_{j}\right)} \|\right|<\varepsilon_{n} .
$$

Definition 2.6. Let $X$ be a separable infinite-dimensional Banach space. A normalized basic sequence $\left(x_{i}\right) \subset X$ generates a spreading model $\left(\tilde{x}_{i}\right)$ if for some $\varepsilon_{n} \downarrow 0$, for all $n \in N, n \leq k_{1}<$ $\cdots<k_{n}$, and $\left(a_{i}\right)_{1}^{n} \subseteq[-1,1]$,

$$
\left(1+\varepsilon_{n}\right)^{-1}\left\|\sum_{i=1}^{n} a_{i} \tilde{x}_{i}\right\| \leq\left\|\sum_{i=1}^{n} a_{i} x_{k_{i}}\right\| \leq\left(1+\varepsilon_{n}\right)\left\|\sum_{i=1}^{n} a_{i} \tilde{x}_{i}\right\| .
$$

Theme 2.7. Definition 2.6 is equivalent to Definition 2.1.

Proof. We can easily conclude Definition 2.1 from Definition 2.6

By the Definition 2.1, we know that $\left(\tilde{x}_{i}\right)$ is a spreading model generated by $\left(x_{i}\right)$. For any fixed $\left(a_{i}\right)_{i=1}^{n} \subseteq[-1,1]$, we partition $[0, n]$ into some subintervals $\left(I_{j}\right)_{j=1}^{m}$ of length $<\varepsilon_{\rho}$ and "color" $\left(k_{1}, k_{2}, \ldots k_{n}\right)$ by $I_{l}$ if

$$
\left\|\sum_{i=1}^{n} a_{j} y_{k_{i}}\right\| \in I_{l} \quad(1 \leq l \leq m) .
$$

Let $\rho \in Z^{+}, \rho \geq n$ and $\rho \leq k_{1}<\cdots<k_{i_{0}}<\cdots k_{n}$; then

$$
\left\|\left|\left\|\sum_{i=1}^{n} a_{i} x_{k_{i}}\right\|-\left\|\sum_{i=1}^{n} a_{i} \tilde{x}_{i}\right\|\right|<\delta_{\rho},\right.
$$


where $\delta_{\rho} \downarrow 0, \delta_{\rho}>0$. Using the same procedure of Theorem 2.2, we can get that for any $\left(a_{i}\right)_{i=1}^{n} \subseteq[-1,1], \varepsilon_{n} \downarrow 0$,

$$
\left\|\left|\sum_{i=1}^{n} \frac{1}{1+\varepsilon_{n}} a_{i} x_{k_{i}}\|-\| \sum_{i=1}^{n} \frac{1}{1+\varepsilon_{n}} a_{i} \tilde{x}_{i} \|\right|<\delta_{\rho} .\right.
$$

Thus

$$
\left\|\sum_{i=1}^{n} \frac{1}{1+\varepsilon_{n}} a_{i} x_{k_{i}}\right\|<\delta_{\rho}+\left\|\sum_{i=1}^{n} \frac{1}{1+\varepsilon_{n}} a_{i} \tilde{x}_{i}\right\|=\delta_{\rho}+\frac{1}{1+\varepsilon_{n}}\left\|\sum_{i=1}^{n} a_{i} \tilde{x}_{i}\right\| \leq \delta_{\rho}+\left\|\sum_{i=1}^{n} a_{i} \tilde{x}_{i}\right\| .
$$

Letting $\rho \rightarrow \infty$, then

$$
\left\|\sum_{i=1}^{n} \frac{1}{1+\varepsilon_{n}} a_{i} x_{k_{i}}\right\| \leq\left\|\sum_{i=1}^{n} a_{i} \tilde{x}_{i}\right\| .
$$

That is,

$$
\left\|\sum_{i=1}^{n} a_{i} x_{k_{i}}\right\| \leq\left(1+\varepsilon_{n}\right)\left\|\sum_{i=1}^{n} a_{i} \tilde{x}_{i}\right\|
$$

Similarly,

$$
\left(1+\varepsilon_{n}\right)^{-1}\left\|\sum_{i=1}^{n} a_{i} \tilde{x}_{i}\right\| \leq\left\|\sum_{i=1}^{n} a_{i} x_{k_{i}}\right\|
$$

Hence, we obtain that

$$
\left(1+\varepsilon_{n}\right)^{-1}\left\|\sum_{i=1}^{n} a_{i} \tilde{x}_{i}\right\| \leq\left\|\sum_{i=1}^{n} a_{i} x_{k_{i}}\right\| \leq\left(1+\varepsilon_{n}\right)\left\|\sum_{i=1}^{n} a_{i} \tilde{x}_{i}\right\| .
$$

Let $S P_{w}(X)$ be the set of all spreading models $\left(\tilde{x}_{i}\right)$ generated by weakly null sequences $\left(x_{i}\right)$ in $X$ endowed with order relation by domination, that is, $\left(\tilde{x}_{i}\right) \leq\left(\tilde{y}_{i}\right)$ if there exists a constant $K \geq 1$ such that $\left\|\sum a_{i} \tilde{x}_{i}\right\| \leq K\left\|\sum a_{i} \tilde{y}_{i}\right\|$ for scalars $\left(a_{i}\right)$; then $\left(S P_{w}(X), \leq\right)$ is a partial order set. If $\left(\tilde{x}_{i}\right) \leq\left(\tilde{y}_{i}\right)$ and $\left(\tilde{y}_{i}\right) \leq\left(\tilde{x}_{i}\right)$, we call $\left(\tilde{x}_{i}\right)$ equivalent to $\left(\tilde{y}_{i}\right)$, denoted by $\left(\tilde{x}_{i}\right) \sim\left(\tilde{y}_{i}\right)$. We identify $\left(\tilde{x}_{i}\right)$ and $\left(\tilde{y}_{i}\right)$ in $S P_{w}(X)$ if $\left(\tilde{x}_{i}\right) \sim\left(\tilde{y}_{i}\right)$.

Lemma 2.8 (see [5]). If an Orlicz sequence space $h_{M}$ does not contain an isomorphic copy of $l_{1}$, then the sets $S P_{w}\left(h_{M}\right)$ and $C_{M, 1}$ coincide. That is, $S P_{w}\left(h_{M}\right)=C_{M, 1}$. 


\section{Orlicz Sequence Spaces with Equivalent Spreading Models}

Definition 3.1 (see [7]). Let $\left(x_{n}\right)$ be a normalized Schauder basis of a Banach space $X .\left(x_{n}\right)$ is said to be lower (resp., upper) semihomogeneous if every normalized block basic sequence of the basis dominates (resp., is dominated by) $\left(x_{n}\right)$.

Lemma 3.2 (see [7]). Let $M$ be an Orlicz function with $M(1)=1, M \in \Delta_{2}$, and let $\left(e_{i}\right)$ denote the unit vector basis of the space $h_{M}$. The basis is

(a) lower semi-homogeneous if and only if $C M(s t) \geq M(s) M(t)$ for all $s, t \in[0,1]$ and some $C \geq 1$,

(b) upper semi-homogeneous if and only if $M(s t) \leq C M(s) M(t)$ for $s, t, C$ as above.

Lemma 3.3 (see [6]). The space $l_{p}$, or $c_{0}$ if $p=\infty$, is isomorphic to a subspace of an Orlicz sequence space $h_{M}$ if and only if $p \in\left[\alpha_{M}, \beta_{M}\right]$, where

$$
\begin{gathered}
\alpha_{M}=\sup \left\{q: \sup _{\substack{0<\lambda, t \leq 1}} \frac{M(\lambda t)}{M(\lambda) t^{q}}<\infty\right\}, \\
\beta_{M}=\inf \left\{q: \sup _{\substack{0<\lambda, t \leq 1}} \frac{M(\lambda t)}{M(\lambda) t^{q}}>0\right\} .
\end{gathered}
$$

Lemma 3.4 (see [5]). Let $M \in \Delta_{2}, l_{M}$ be an Orlicz sequence space which is not isomorphic to $l_{1}$. Suppose that $S P_{w}\left(l_{M}\right)$ is countable, up to equivalence. Then

(i) the unit vector basis of $l_{M}$ is the upper bound of $S P_{w}\left(l_{M}\right)$;

(ii) the unit vector basis of $l_{p}$ is the lower bound of $S P_{w}\left(l_{M}\right)$, where $p \in\left[\alpha_{M}, \beta_{M}\right]$.

Theorem 3.5. Let $M \in \Delta_{2}$, and let $\left(e_{i}\right)$ be the unit basis of the space $l_{M}$. If $\left(e_{i}\right)$ is lower semihomogeneous, then $\left|S P_{w}\left(l_{M}\right)\right|=1$ if and only if $l_{M}$ is isomorphic to $l_{p}, p \in\left[\alpha_{M}, \beta_{M}\right]$.

Proof. Sufficiency. Since $M \in \Delta_{2}, S P_{w}\left(l_{M}\right)$ is countable, then by Lemma 3.4, $l_{M}$ is the upper bound of $S P_{w}\left(l_{M}\right)$, and $l_{p}, p \in\left[\alpha_{M}, \beta_{M}\right]$ is the lower bound of $S P_{w}\left(l_{M}\right)$. Since $l_{M}$ is isomorphic to $l_{p}, p \in\left[\alpha_{M}, \beta_{M}\right]$, we get $\left|S P_{w}\left(l_{M}\right)\right|=1$.

Necessity. If $\left|S P_{w}\left(l_{M}\right)\right|=1$, then $\left|C_{M, 1}\right|=1$ by Lemma 2.8 , that is, all the functions in $C_{M, 1}$ are equivalent to $M$.

For $p \in\left[\alpha_{M}, \beta_{M}\right]$, we define the function $M_{n}(t)[6]$ as follows:

$$
M_{n}(t)=A_{n}^{-1} \int_{u_{n} / \omega_{n}}^{1} M\left(t s \omega_{n}\right) s^{-p-1} d s
$$


where $0<u_{n}<v_{n}<\omega_{n} \leq 1$ with $\omega_{n} \rightarrow 0, u_{n} / v_{n} \rightarrow 0, A_{n}=\int_{u_{n} / \omega_{n}}^{1} M\left(s \omega_{n}\right) s^{-p-1} d s$. Obviously, $M_{n}(t) \in C_{M, 1}$; next we shall prove that $M_{n}(t)$ is equivalent to $M$

$$
\frac{M_{n}(t)}{M(t)}=A_{n}^{-1} \int_{u_{n} / w_{n}}^{1} \frac{M\left(t s w_{n}\right)}{M(t)} s^{-p-1} d s
$$

Since $s \leq 1, s w_{n} \leq w_{n}$, and $M$ is nondecreasing convex function, therefore, $M\left(t s w_{n}\right) \leq$ $M\left(t w_{n}\right)$; then

$$
\begin{aligned}
\frac{M_{n}(t)}{M(t)} & =A_{n}^{-1} \int_{u_{n} / w_{n}}^{1} \frac{M\left(t s w_{n}\right)}{M(t)} s^{-p-1} d s \\
& \leq A_{n}^{-1} \int_{u_{n} / w_{n}}^{1} \frac{M\left(t w_{n}\right)}{M(t)} s^{-p-1} d s \\
& =\frac{1}{p} A_{n}^{-1} \frac{M\left(t w_{n}\right)}{M(t)}\left(1-\left(\frac{u_{n}}{w_{n}}\right)^{-p}\right) .
\end{aligned}
$$

Since $t w_{n}<t$ and $M\left(t w_{n}\right)<M(t)$, we have

$$
\frac{M_{n}(t)}{M(t)} \leq A_{n}^{-1} \frac{M\left(t w_{n}\right)}{M(t)}\left(1-\left(\frac{u_{n}}{w_{n}}\right)^{-p}\right) \leq \frac{1}{p} A_{n}^{-1}\left(1-\left(\frac{u_{n}}{w_{n}}\right)^{-p}\right)
$$

Notice that for any fixed $n$, the right side of the above inequality is a constant; then we obtain $M_{n} \leq M$

$$
\frac{M_{n}(t)}{M(t)}=A_{n}^{-1} \int_{u_{n} / w_{n}}^{1} \frac{M\left(t s w_{n}\right)}{M(t)} s^{-p-1} d s
$$

By $u_{n} / w_{n} \leq s \leq 1$, we have $s^{-p-1} \geq\left(u_{n} / w_{n}\right)^{-p-1}$ and $M\left(t s w_{n}\right) \geq M\left(t u_{n}\right)$; hence

$$
\frac{M_{n}(t)}{M(t)} \geq A_{n}^{-1} \frac{M\left(t u_{n}\right)}{M(t)}\left(\frac{u_{n}}{w_{n}}\right)^{-p-1}\left(1-\frac{u_{n}}{w_{n}}\right)
$$

Since $\varphi(t)=M(t) / t^{p}, n \varphi\left(w_{n}\right)<\varphi\left(v_{n} / 2\right)$, and

$$
\frac{n M\left(u_{n}\right)}{w_{n}^{p}}<\frac{M\left(v_{n} / 2\right)}{\left(v_{n} / 2\right)^{p}}
$$

Moreover,

$$
\frac{w_{n}^{p}}{v_{n}^{p}}>\frac{n 2^{-p} M\left(w_{n}\right)}{M\left(v_{n} / 2\right)}
$$


We obtain that

$$
\begin{aligned}
\frac{M_{n}(t)}{M(t)} & \geq A_{n}^{-1} \frac{M\left(t u_{n}\right)}{M(t)}\left(\frac{u_{n}}{w_{n}}\right)^{-p-1}\left(1-\frac{u_{n}}{w_{n}}\right) \\
& >A_{n}^{-1}\left(1-\frac{u_{n}}{w_{n}}\right) \frac{w_{n}^{p}}{v_{n}^{p}} \frac{M\left(t u_{n}\right)}{M(t)} \\
& >n \cdot 2^{-p} A_{n}^{-1}\left(1-\frac{u_{n}}{w_{n}}\right) \frac{M\left(w_{n}\right)}{M\left(v_{n} / 2\right)} \frac{M\left(t u_{n}\right)}{M(t)}
\end{aligned}
$$

Since $0<t, u_{n} \leq 1,\left\{e_{i}\right\}$ is lower semihomogeneous; then by Lemma 3.2, we have for some $C \geq 1$

$$
C M\left(t u_{n}\right) \geq M(t) M\left(u_{n}\right)
$$

Therefore,

$$
\frac{M_{n}(t)}{M(t)}>n \cdot 2^{-p} C^{-1} A_{n}^{-1}\left(1-\frac{u_{n}}{w_{n}}\right) \frac{M\left(w_{n}\right)}{M\left(v_{n} / 2\right)} M\left(u_{n}\right)
$$

Thus we get $M_{n} \geq M$.

So by (3.4) and (3.7), we can know that $M_{n}$ is equivalent to $M$. By Lemma 3.3 and its proof ([6], Theorem 4.a.9), we obtain that $M_{n}(t)$ uniformly converges to $t^{p}$ on $[0,1 / 2]$. Since $C_{M, 1}$ is the closed subset of $C[0,1 / 2]$, we have that $t^{p} \in C_{M, 1}, t^{p}$ is equivalent to $M$, and therefore $l_{M}$ is isomorphic to $l_{p}$.

\section{Acknowledgment}

The first author was supported by the NSF of China (no. 10671048) and by Haiwai Xueren Research Foundation in Heilongjiang Province (no. 1055HZ003).

\section{References}

[1] E. Odell, On the Structure of Separable Infinite Dimensional Banach Spaces, Lecture Notes in Chern Institute of Mathematics, Nankai University, Tianjin, China, 2007.

[2] G. Androulakis, E. Odell, Th. Schlumprecht, and N. Tomczak-Jaegermann, "On the structure of the spreading models of a Banach space," Canadian Journal of Mathematics, vol. 57, no. 4, pp. 673-707, 2005.

[3] S. J. Dilworth, E. Odell, and B. Sari, "Lattice structures and spreading models," Israel Journal of Mathematics, vol. 161, pp. 387-411, 2007.

[4] A. Brunel and L. Sucheston, "On B-convex Banach spaces," Mathematical Systems Theory, vol. 7, no. 4, pp. 294-299, 1974.

[5] B. Sari, "On the structure of the set of symmetric sequences in Orlicz sequence spaces," Canadian Mathematical Bulletin, vol. 50, no. 1, pp. 138-148, 2007.

[6] J. Lindenstrauss and L. Tzafriri, Classical Banach Spaces, vol. 1, Springer, New York, NY, USA, 1977.

[7] M. González, B. Sari, and M. Wojtowicz, "Semi-homogeneous bases in Orlicz sequence spaces," in Function Spaces, vol. 435 of Contemporary Mathematics, pp. 171-181, American Mathematical Society, Providence, RI, USA, 2007. 